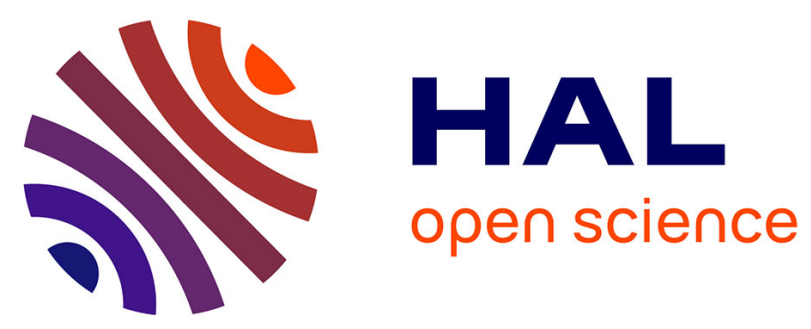

\title{
LA RELAXATION DANS LES GAZ \\ VISCO-THERMIQUES : COMPARAISON ENTRE THÉORIE ET EXPÉRIENCE
}

J. Polack, S. Nezan

\section{> To cite this version:}

J. Polack, S. Nezan. LA RELAXATION DANS LES GAZ VISCO-THERMIQUES : COMPARAISON ENTRE THÉORIE ET EXPÉRIENCE. Journal de Physique IV Proceedings, 1992, 02 (C1), pp.C1801-C1-804. 10.1051/jp4:19921175 . jpa-00251136

\section{HAL Id: jpa-00251136 https://hal.science/jpa-00251136}

Submitted on 1 Jan 1992

HAL is a multi-disciplinary open access archive for the deposit and dissemination of scientific research documents, whether they are published or not. The documents may come from teaching and research institutions in France or abroad, or from public or private research centers.
L'archive ouverte pluridisciplinaire HAL, est destinée au dépôt et à la diffusion de documents scientifiques de niveau recherche, publiés ou non, émanant des établissements d'enseignement et de recherche français ou étrangers, des laboratoires publics ou privés. 


\title{
LA RELAXATION DANS LES GAZ VISCO-THERMIQUES : COMPARAISON ENTRE THÉORIE ET EXPÉRIENCE
}

\author{
J.D. POLACK et S. NEZAN
}

Laboratoire d'Acoustique de l'Université du Maine, CNRS - URA 1101, BP. 535, F-72017 Le Mans cedex, France

Resumé : Le formalisme classique de la propagation sonore dans les gaz visco-thermiques est modifié pour inclure la relaxation. Nous en déduisons l'expression complexe du coefficient d'atténuation, que nous retrouvons expérimentalement.

\begin{abstract}
The classical formalism for sound propagation in thermoviscous gases is modified to include relaxation. A complex attenuation coefficient is then deduced and verified experimentally.
\end{abstract}

\section{Introduction.}

L'approche temporelle de la propagation des ondes sonores dans les gaz visco-thermiques se révèle très féconde si l'on veut comprendre la nature des phénomènes en jeu. Jusqu'à présent $/ 1 /$, cette formulation a négligé les phénomènes de relaxation. c'est donc à la relaxation, et à la manière dont elle modifie l'équation de Kirchhoff, que s'intéresse le présent travail.

\section{Le formalisme en l'absence de relaxation.}

En 1 'absence de relaxation $/ 1 /$, les équations de départ sont les trois équations de conservation, complétées par deux équations d'état :

- l'équation de continuité :

$$
\partial_{t} \rho+\rho_{0} d i v v=q_{s},
$$

- I'équation de stokes-Navier :

$$
\partial_{t} v+\left(1 / \rho_{0}\right) \nabla p=c I_{v} \nabla d i v v-c I_{v}^{\prime} \operatorname{rotxot} v,
$$

- la conservation de l'énergie, qui se réduit à :

$$
\rho_{0} T \partial_{t} s=\rho_{0} C C_{p} I_{h} \Delta \tau+r_{s} \text {. }
$$

- les équations d'état qui expriment $p$ et $s$ en fonction des deux variables indépendantes $\rho$ et $\tau$ : 


$$
\begin{gathered}
p=\beta \tau+\left(c^{2} / \gamma\right) \rho, \\
s=\left(C_{v} / T\right) \tau-\left[c^{2} /\left(\rho_{0} \gamma T\right)\right] \rho .
\end{gathered}
$$

$l_{\mathrm{v}}, l_{\mathrm{v}}^{\prime}$ et $l_{\mathrm{h}}$ sont des longueurs caractéristiques visqueuses et thermiques. Les autres notations suivent la référence $/ 1 /$.

\section{La relaxation dans l'air.}

Dans le cadre de l'acoustique linéaire, la relaxation ne modifie pas les équations de conservation $/ 2 /$, car le taux de production irréversible d'entropie est une quantité du deuxième ordre. Par contre, les équations d'état sont modifiées.

Dans l'air, toutes les réactions de relaxations conservent le même nombre de môles de gaz /3/. La pression ne change donc pas pour une réaction isotherme et isochore /2/, c'est-à-dire pour notre choix de variables thermodynamiques : I'équation (4) reste donc valable.

Le calcul montre alors que seule la contribution thermique à l'entropie est perturbée par la relaxation $/ 4 /$. L'équation d'état (5) s'écrit alors, au sens des opérateurs (l'indice $i$ décrit les différentes équations de relaxation) :

$$
s=\left(\frac{C_{V}}{T}-\sum_{i} \frac{C_{i}}{T} \frac{\theta_{i} \partial_{t}}{1+\theta_{i} \partial_{t}}\right) \tau-\frac{C^{2}}{\rho_{0} \gamma T} \rho,
$$

Tout se passe alors comme si l'on remplaçait la chaleur spécifique à volume constant $c_{v}$ par 1 'opérateur :

$$
\tilde{C}_{v}=C_{v}-\sum_{i} c_{i} \frac{\theta_{i} \partial_{t}}{1+\theta_{i} \partial_{t}}
$$

ce qui permet d'introduire l'opérateur de relaxation $U_{v}$ par :

$$
U_{v}=\frac{\tilde{C}_{v}}{C_{v}}=1-\sum_{i} \frac{\gamma}{\gamma_{i}} \frac{\theta_{i} \partial_{t}}{1+\theta_{i} \partial_{t}}
$$

où $\gamma_{i}=C_{p} / C_{i}$. De la même manière, l'opérateur $U_{p}$ s'écrit :

$$
U_{p}=\frac{\tilde{C}_{p}}{C_{p}}=1-\sum_{i} \frac{1}{\gamma_{i}} \frac{\theta_{i} \partial_{t}}{1+\theta_{i} \partial_{t}} \text {. }
$$

L'equation de Kirchhoff prend alors la forme:

$$
\left\{\partial_{t}^{3}-\left[c^{2} U_{p} U_{v}^{-1}+c\left(I_{v}+\gamma I_{h} U_{v}^{-1}\right) \partial_{t}\right] \partial_{t} \Delta+\left[c^{2} I_{h} U_{v}^{-1}\left(c+\gamma I_{v} \partial_{t}\right)\right] \Delta^{2}\right\} \phi=\text { Sources }
$$

qui peut se factoriser de la manière habituelle en une équation thermique et une équation acoustique $/ 1 /$. 
4. Le coefficient d'attenuation sonore.

La résolution de l'équation acoustique introduit alors le coefficient d'atténuation :

$$
\alpha(f)=-j \frac{\omega}{c}\left[1-\left(\frac{U_{v}}{U_{p}+j\left[U_{v} I_{v}+(\gamma-1) U_{p}^{-1} I_{h}\right] \frac{\omega}{C}}\right)^{1 / 2}\right],
$$

où $\omega=2 \pi f$ est la pulsation, et où $U_{v}$ et $U_{p}$ sont maintenant donnés dans le domaine fréquentiel par:

$$
U_{v}=1-\sum_{i} \frac{\gamma}{\gamma_{i}} \frac{j \omega \theta_{i}}{1+j \omega \theta_{i}} \quad, \quad U_{p}=1-\sum_{i} \frac{1}{\gamma_{i}} \frac{j \omega \theta_{i}}{1+j \omega \theta_{i}} .
$$

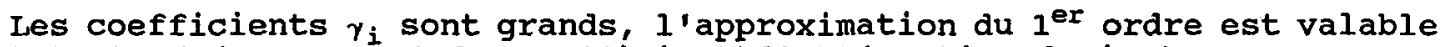
à toute fréquence, et le coefficient d'atténuation devient :

$$
\alpha(f)=\frac{1}{2}\left(\frac{2 \pi f}{c}\right)^{2}\left[I_{v}+(\gamma-1) I_{h}+\sum_{i} \frac{\gamma-1}{\gamma_{i}} \frac{c /\left(2 \pi f_{r i}\right)}{1+j f / f_{r i}}\right],
$$

où les $f_{r i}=1 /\left(2 \pi \theta_{i}\right)$ sont les fréquences de relaxation. C'est donc un nombre complexe, dont la partie imaginaire correspond au déphasage introduit par la relaxation.

\section{La mesure.}

L'atténuation se déduit du rapport des pressions sonores mesurées par deux microphones (B\&K $4138,1 / 8 ")$ à différentes distances d'une même source. Mais pour nous affranchir de la réponse des microphones, nous avons conservé un microphone de référence $\mathrm{M}_{2}$ à distance fixe de la source $(0,4 \mathrm{~m})$, tandis que le microphone de mesure $M_{1}$ se déplace de 0,4 à $9,4 \mathrm{~m}$ par pas de $1 \mathrm{~m}$ (fig. 1 ).

D'autre part, pour pouvoir discriminer le son direct des éventuelles réflexions, nous avons choisi une source impulsive, créée par une décharge électrique. Il en résulte une onde de pression très brève (moins de $50 \mu s$ à toutes distances), et la possibilité de conduire les mesures dans une salle non-sourde, à condition de s'éloigner suffisamment des parois. En outre, en additionnant plusieurs

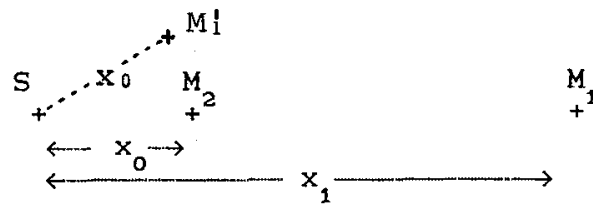

Figure 1 : Le principe de mesure. ondes de manière cohérente, le bruit de fond peut être réduit de manière importante.

Le calcul de l'atténuation résulte d'un simple traitement des signaux recueillis (estimation de la fonction de transfert complexe). II est effectué sur station de travail MC5450 (Concurrent Computer Corporation) à l'aide du logiciel ILs. Il se complique un peu du fait qu'il s'effectue en deux temps : le calcul de 1'atténuation entre les deux microphones situés à des distances différentes est corrigé par la mesure d'étalonnage effectuée avec les deux microphones situés à même distance de la source. Ainsi l'influence de différences entre les deux microphones est-elle 
minimisée, ainsi que celle des inévitables fluctuations d'intensité de la source impulsionnelle.

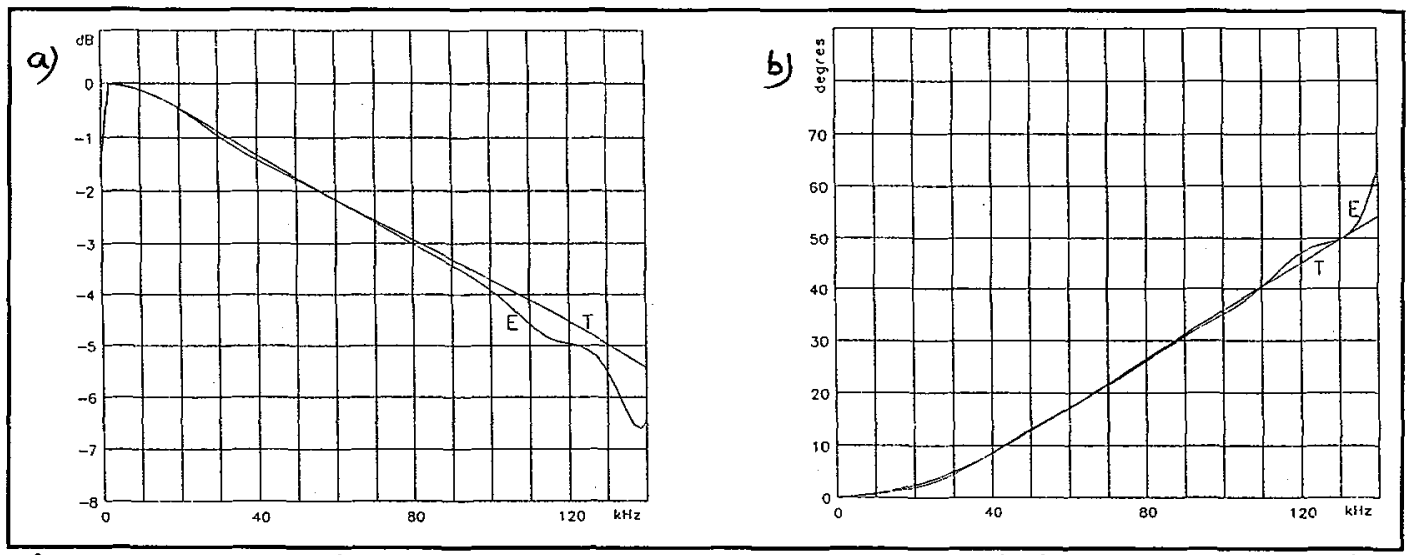

Figure 2 : Amplitude (a) et phase (b) du coefficient d'atténuation évaluées sur $5 \mathrm{~m}$. Théorie (T) et expérience (E).

La figure 2 présente l'atténuation ramenée à $1 \mathrm{~m}$ pour une distance de 5 m entre les microphones (courbe E). Par rapport à l'équation (13) (courbe $T$ ), on note une bonne adéquation de la phase jusqu'à $110 \mathrm{kHz}$, et de l'amplitude jusqu'à $100 \mathrm{kHz}$. Par contre, aux fréquences extrêmes, le signal sonore $n$ 'émerge plus suffisamment du bruit de fond pour autoriser une estimation correcte de l'atténuation. Mais la phase qui reste correcte confirme la validité de la théorie à ces fréquences.

Les oscillations de la courbe expérimentale ne se retrouvent pas sur la courbe théorique. L'analyse des sources d'erreurs révèle que les diffractions sur les microphones et sur le bord de la source suffisent à créer ces fluctuations. En effet, dans l'idée de s'affranchir d'une éventuelle directivité de la source, les deux microphones sont placés en ligne avec la source, sauf pour la mesure d'étalonnage (cf. fig. 1). Mais l'expérience a prouvé qu'une simple manipulation d'un microphone sur son support suffit à créer des variations de l'ordre du demi-décibel, c'està-dire supérieures à la précision recherchée.

\section{Conclusion.}

Les résultats expérimentaux ne laissent aucun doute sur la validité de l'équation (13) du coefficient d'atténuation complexe. De plus, dans la plupart des applications (e.g. /5/), ni les fluctuations de la source, ni les perturbations apportées par le microphone, ne peuvent être compensées, ce qui se traduit par une incertitude de mesure bien supérieure à celle de la théorie. Il est donc tout à fait justifié de retenir l'équation (13) pour compenser l'atténuation visco-thermique.

/1/ POLACK, J-D, J. d'Acoust. 4 (1991) 47

/2/ BAUER, HJ, "Physical Acoustics", vol. IIA, édité par Mason \& Thurston (Academic Press, 1965)

/3/ BASS, HE, SUTHERLAND, LC, PIERCY,J, EVANS, L, "Physical Acoustics" vol.XVII, édité par Mason \& Thurston (Ácademic Press, 1984)

/4/ PIERCE, AD, Z. angew. Math. Mech. 58 (1978) T297

/5/ MEYNIAL, $X$, POLACK,J-D, DODD, G, 2ème CFA, Arcachon, 1992 